\title{
Extensive Fiber Comparison and GN-model Validation in Uncompensated Links using DAC-generated Nyquist-WDM PM-16QAM Channels
}

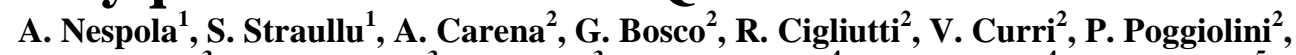

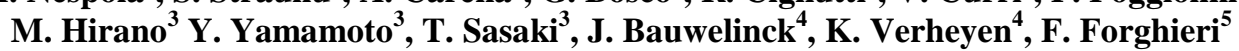

${ }^{1}$ Istituto Superiore Mario Boella, via Pier Carlo Boggio 61, 10138 Torino, Italy

${ }^{2}$ Dipartimento di Elettronica e Telecomunicazioni, Politecnico di Torino, corso Duca degli Abruzzi, 24,10129 Torino, Italy

${ }^{3}$ Sumitomo Electric Industries, LTD, 1, Taya-cho, Sakae-ku, Yokohama, 244-8588, Japan

${ }^{4}$ INTEC/IMEC, Ghent University, Sint-Pietersnieuwstraat 41, 9000 Ghent, Belgium

${ }^{5}$ Cisco Photonics Italy srl, via Philips 12, 20900 Monza, Italy

andrea.carena@polito.it

\begin{abstract}
We report on a comprehensive performance comparison of seven different fiber types: transmission experiments based on of Nyquist-WDM using PM-16QAM modulation over uncompensated links show a perfect agreement with GN model prediction.

OCIS codes: (060.0060) Fiber optics and optical communications; (060.1660) Coherent communications;
\end{abstract}

\section{Introduction}

With the development of coherent detection based on digital signal processing (DSP), optical communication systems are evolving towards the use of multilevel modulation formats to increase the available capacity. For this class of systems, it has been demonstrated that optimal performance is achieved in the absence of optical dispersion management [1]. Under these conditions, it has been shown that fiber nonlinearities act introducing an additional Gaussian Noise-like component called nonlinear interference (NLI): the theory, called GN-model, allowing to analytically derive the amount of NLI has been described and validated by simulations and experiments [2].

In this paper we report an experimental comprehensive performance comparison of several fiber types, based on the evaluation of the maximum reach over a multi-span optical system with EDFA-only amplification of a NyquistWDM signal [3] composed of 22 PM-16QAM sub-channels at $125 \mathrm{~Gb} / \mathrm{s}$. The PM-16QAM sub-channels were generated through digital signal processing (DSP) and digital to analog converters (DACs), with a frequency spacing equal to 1.024 times the symbol-rate. We carried out measurements on seven different fiber types: four pure-silica core fibers (PSCF) with different effective area, a standard SSMF, a NZDSF and a dispersion compensating fiber (DCF). DCF, used here as a transmission fiber, was tested for the purpose of extending the experimental GN-model validation to a much broader parameters set. Moreover, we clearly show the role of high local dispersion in the mitigation of nonlinear effects.

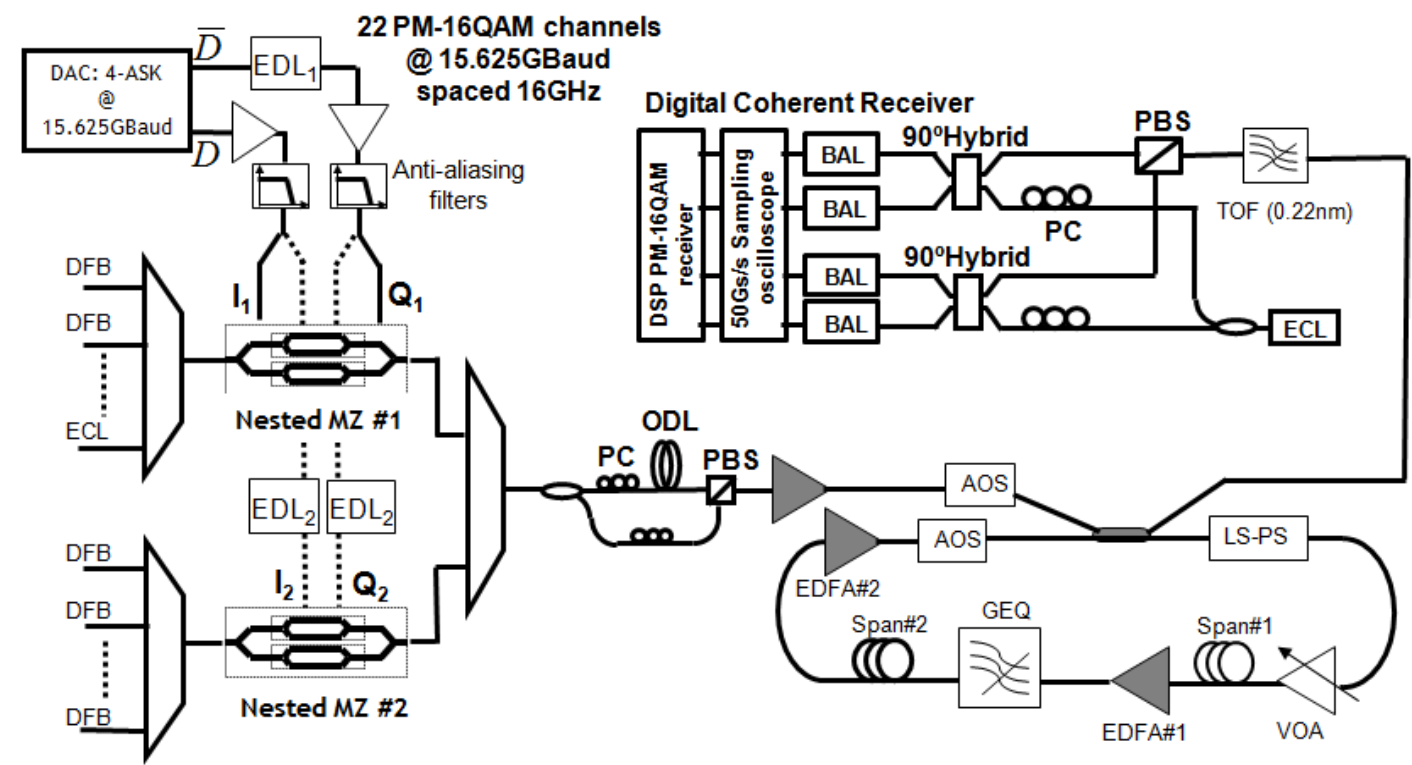

Fig. 1: Experimental setup. 


\section{Experimental setup}

The experimental setup is shown in Fig. 1. An array of 22 lasers positioned between $1555.930 \mathrm{~nm}$ and $1558.648 \mathrm{~nm}$ was finely tuned at $16 \mathrm{GHz}$ frequency separation. The channel under test, at the center of the comb, was generated by an external cavity laser (ECL) while for all interfering channels we used distributed-feedback (DFB) lasers. The odd and even carriers were separately fed to two distinct nested Mach-Zehnder modulators (NMZM). The electrical signals driving the NMZMs were obtained as follows. Two distinct $2^{15}-1$ pseudo-random binary sequences (PRBSs) were generated on a computer and were combined to form a 4-level amplitude-shift keying (ASK) signal with nonreturn-to-zero symbols. The 4-ASK signal was then digitally filtered to give it a sharp square-root raised-cosine spectral shape, with roll-off 0.01 and electrical bandwidth equal to half the symbol rate. The samples of the filtered 4-ASK signal were then fed to an arbitrary waveform generator (AWG) Tektronix 7122B running at 23.4375 GS/s, i.e., at 1.5 samples per symbol. The I and Q driving signals for the even-channels NMZM were obtained from the two complementary outputs of the DAC, with the insertion of 85 symbols delay on the quadrature arm for decorrelation (electrical delay line, EDL1 in Fig. 1). Both in-phase and quadrature electrical signals were further split and delayed (EDL2 in Fig. 1) by 134 symbols to generate the driving signals for the odd-channel NMZM. The NMZMs were driven in the linear region of their electro-optic characteristic (the modulation depth on I and Q arms for both the NMZMs was approximately $25 \%$ ). The frequency response of the overall electrical chain was precompensated for in the DSP at Tx, in order to obtain an electrical power spectrum at the input of the modulator characterized by flat top and sharp cut-off. The spurious replica of the spectrum caused by the finite sampling rate of the AWG were attenuated using ad-hoc anti-aliasing electrical filters provided by IMEC. Finally, even and odd channels were optically coupled with a polarization maintaining $3 \mathrm{~dB}$ coupler. The resulting 22-channel singlepolarization optical signal was split and recombined onto two orthogonal polarizations for PM emulation, with one polarization going through $64 \mathrm{~m}$ of a fiber optical-delay-line (ODL in Fig. 1) for decorrelation. No optical filtering was applied at the transmitter side.

The WDM signal was launched into a re-circulating fiber loop consisting of two spans of the fiber under test. A loop synchronous polarization scrambler (LS-PS) was inserted to emulate the polarization changes occurring in a longhaul transmission link. The loop made use of EDFA-only amplification and included a gain-equalizer (GEQ). The equivalent noise figure was measured to be about $5.5 \mathrm{~dB}$. The parameters for all seven investigated fibers are listed in detail in Table 1: all values are taken directly from manufacturer or measured directly on the spool. $\alpha, \gamma$ and $\mathrm{D}$ are the fiber loss, dispersion and non-linearity coefficients, respectively. Extra-losses due to splices with SSMF patchcords, not negligible when differences in effective area are substantial, have been carefully characterized in order to be taken into account in model prediction. PSCF80 (Z-fiber ${ }^{\circledR}$ ), PSCF110 (Z-plus fiber ${ }^{\circledR}$ ), PSCF130 (Z++), PSCF150 and DCF were supplied by Sumitomo Electric Industries Ltd.

The optical receiver had a standard set-up for coherent reception, with a tunable external-cavity laser (ECL) as local oscillator (LO) and a standard optical hybrid for signal and LO mixing. Further details can be found in [3]. Tx and LO lasers were two distinct ECLs, with a linewidth of less than $100 \mathrm{kHz}$ each. At the Rx input, a tunable optical filter (TOF) with bandwidth $0.22 \mathrm{~nm}$ was inserted to prevent excessive optical power from reaching the photodetectors. The off-line receiver DSP consisted of the following functional blocks. First, a re-sampling stage lowered the sample rate from 3.2 samples per symbol down to 2 . Then, a first equalizer stage performed bulk CD compensation, followed by a multiple-input, multiple-output (MIMO) equalizer stage, adjusted through a multimodulus constant modulus algorithm (MM-CMA). Frequency offset estimation and compensation was then performed through a Viterbi\&Viterbi stage, modified to properly filter out the 16QAM modulation [4]. Finally, minimum distance decision was performed.

\begin{tabular}{|c|c|c|c|c|c|c|}
\hline Fiber & $\begin{array}{c}\boldsymbol{\alpha} \\
{[\mathbf{d B} / \mathbf{k m}]}\end{array}$ & $\begin{array}{c}\boldsymbol{\gamma} \\
{[\mathbf{1} / \mathbf{W} / \mathbf{k m}]}\end{array}$ & $\begin{array}{c}\mathbf{D} \\
{[\mathbf{p s} / \mathbf{n m} / \mathbf{k m}]}\end{array}$ & $\begin{array}{c}\text { Span Length }-\mathbf{L}_{\text {span }} \\
{[\mathbf{k m}]}\end{array}$ & $\begin{array}{c}\text { Max Reach } \\
{[\# \mathbf{s p a n s}]}\end{array}$ & $\begin{array}{c}\text { Max Reach } \\
{[\mathbf{k m}]}\end{array}$ \\
\hline SSMF & 0.190 & 1.26 & 16.84 & 51.06 & 38 & 12 \\
\hline NZDSF & 0.200 & 2.00 & 2.58 & 50.18 & 44 & 602 \\
\hline PSCF 80 & 0.164 & 1.04 & 16.36 & 54.44 & 58 & 2395 \\
\hline PSCF 110 & 0.161 & 0.81 & 20.50 & 53.18 & 62 & 3084 \\
\hline PSCF 130 & 0.162 & 0.68 & 20.92 & 54.42 & 70 & 3374 \\
\hline PSCF 150 & 0.161 & 0.59 & 20.69 & 54.44 & 25 & 3810 \\
\hline DCF & 0.457 & 6.03 & -166.47 & 20.11 & 502 \\
\hline
\end{tabular}

Table 1: Fiber parameters. 


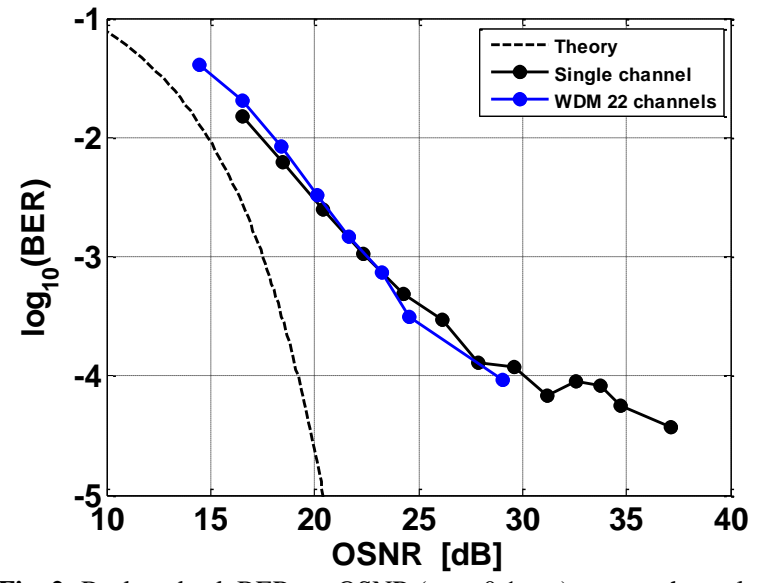

Fig. 2: Back-to-back BER vs. OSNR (over $0.1 \mathrm{~nm}$ ), center channel.

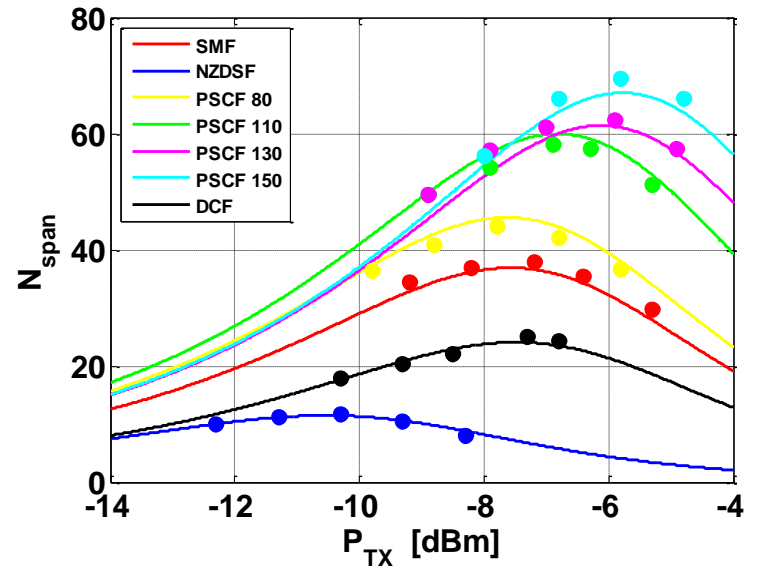

Fig. 3: Reach vs. $\mathrm{P}_{\mathrm{TX}}$ for fibers under analysis: measurements (dots) and GN-model (solid lines)

\section{Results from experiment and GN-model}

All measurements reported were carried out on the channel at the center of the comb, placed at 1557.223. First, a characterization of the back-to-back (btb) performance was performed, followed by maximum reach measurements at a BER threshold equal to $10^{-2}$, achievable with advanced commercial hard FECs with 20.5\% overhead [5]. Results of the btb characterization are shown in Fig.2. The penalty between single-channel and WDM transmission is negligible: considering the quasi-Nyquist spacing this confirms that DAC-enabled spectral engineering effectively limits inter-channel linear crosstalk, thus allowing the use of extremely tight channel spacing.

Then, for each fiber type, BER measurements were performed varying the fiber launch power and the number of recirculations. Fig. 3 shows, for each fiber and each launch power, the maximum number of fiber spans at which the FEC threshold was not exceeded. In Fig. 3, we also report results obtained using the GN-model [2]: using fiber and system parameters reported in previous section, without any fitting parameter, we were able to accurately predict the reach at each input power. The good agreement between theory and experiment confirms the capability of the model to effectively predict the non-linear effects perturbing the signal propagation along the link.

\section{Comments and conclusions}

We have reported an extensive analysis of fiber performance: a comparison of seven different fiber types in terms of maximum reach has been performed in uncompensated links based on EDFA-only amplification. The new family of pure-silica core fibers outperform SSMF thanks both to the reduced loss and the non-linear coefficient. Testing of the DCF as a transmission fiber allowed to further validate the GN-model proving the strong potential of this tool over a broad range of fiber parameters. Moreover, such test confirmed the important role of dispersion in improving the performance, as predicted by the GN-model: despite the high non-linear coefficient, the DCF showed a relatively high optimal $\mathrm{P}_{\mathrm{TX}}$ allowing to reach 25 spans.

The experimental results obtained over the highest-performance fiber (PSCF150) demonstrate that a Nyquist-WDM system based on PM-16QAM with a net spectral efficiency of $6.25 \mathrm{~b} / \mathrm{s} / \mathrm{Hz}$ and propagating on an EDFA-only link with span length about $55 \mathrm{~km}$ has a reach of more than $3800 \mathrm{~km}$. This result indicates the combination of PM16QAM and large effective area PSCF as a promising solution for long-haul systems allowing to transport more than 30 Tbps per fiber when fully loading the whole C-band.

We thank OCLARO for the nested Mach-Zehnder modulators and Linkra-TeleOptix for the balanced photodetectors. This work was supported by CISCO Systems within a SRA contract.

\section{References}

[1] V. Curri, P. Poggiolini, A. Carena, F. Forghieri, "Dispersion compensation and mitigation of non-linear effects in $111 \mathrm{~Gb} / \mathrm{s}$ WDM coherent PM-QPSK systems,” IEEE Phot. Tech. Letters, vol. 20, Sept. 2008, pp. 1473-1475.

[2] P. Poggiolini, "The GN Model of non-linear propagation in uncompensated coherent optical systems," J. Lightwave Technol., Early Access, 10.1109/JLT.2012.2217729.

[3] R. Cigliutti, et al., "Ultra-long-haul transmission of $16 \times 112 \mathrm{~Gb} / \mathrm{s}$ spectrally-engineered DAC-generated Nyquist-WDM PM- 16QAM Channels with 1.05x(Symbol-Rate) Frequency Spacing," in Proc. OFC/NFOEC 2012, paper OTh3A.3, Los Angeles (CA), USA, Mar. 2012.

[4] I. Fatadin, D. Ives, S.J. Savory "Blind equalization and carrier phase recovery in a 16-QAM optical coherent system," J. Lightwave Technol., vol. 27, n. 15, pp. 3042-3049, Aug.1 2009.

[5] http://www.vitesse.com/products/product.php?number=VSC9804. 